\title{
Aménagement de bas-fonds, politique de l'aménageur et recompositions foncières. Le cas de Lofing au Burkina Faso
}

\author{
Philippe Lavigne Delville ${ }^{1, *}$ et Justine Robin ${ }^{2}$ \\ ${ }^{1}$ UMR GRED, IRD/UPVM, Montpellier, France \\ ${ }^{2}$ Master Anthropologie du développement durable, Aix-Marseille Université, Marseille, France
}

\begin{abstract}
Résumé - L'aménagement des bas-fonds et des petites vallées inondables peut permettre d'accroître la production agricole et la résilience des agricultures familiales en Afrique soudanienne. Mais les opportunités économiques que ces espaces représentent ne peuvent être saisies que par les acteurs ayant accès à une parcelle en leur sein, ce qui pose la question de la politique de l'aménageur en termes de redistribution des parcelles. Or, la prise en compte des questions foncières lors des projets d'aménagement demeure très inégale. À partir du cas d'un aménagement de bas-fond au sud du Burkina Faso, cet article montre comment le devenir des droits existants sur la zone à aménager et les règles d'accès aux nouvelles parcelles déterminent la distribution des gains et des pertes entre les exploitations, et comment les pratiques des intervenants peuvent créer ou réveiller des conflits ou des tensions, en particulier par rapport à la légitimité des règles de redistribution foncière.
\end{abstract}

Mots clés : aménagement hydro-agricole / bas-fond / foncier / droits coutumiers / projet de développement / conflits fonciers

\begin{abstract}
Lowland development, project policy and land tenure change. The case of Lofing in Burkina Faso. The development of lowlands and small flood valleys can increase both agricultural production and resilience of family farming in Sudanian Africa. But the economic opportunities they represent can only be grasped by the actors having access to a plot. Projects' policy in terms of land redistribution is thus crucial. However, the land issues of development schemes remain poorly addressed. Drawing on the case of a lowland development scheme in southern Burkina Faso, this article shows how the existing rights over the area to be developed and the rules for access to new plots determine inclusion and exclusion from the benefits of the land, and thus the distribution of gains and losses between farms. It also shows that the practices of the project's team may create or awaken conflicts or tensions, particularly regarding the legitimacy of land redistribution rules.
\end{abstract}

Keywords: development scheme / lowland / land tenure / customary rights / development project / land conflicts

\section{Introduction}

Les bas-fonds sont les portions amont des réseaux hydrographiques, avec un lit mineur peu ou pas marqué, et sont submergés une partie de l'année par la concentration des ruissellements de surface et parfois par la remontée des nappes superficielles (Raunet, 1985). Ce sont souvent des espaces pluriusages. Historiquement, en Afrique soudanienne, ils servaient principalement d'espaces de pâturage de saison sèche avec des portions consacrées à la riziculture (souvent féminine) et au maraîchage de saison sèche, où des dynamiques spectaculaires

\footnotetext{
*Auteur de correspondance :

philippe.lavignedelville@ird.fr
}

ont été observées en périurbain ou le long des axes de communication (Lavigne Delville, 1998).

Au Burkina Faso, les politiques d'aménagement de basfonds ont débuté sous le régime de Sankara (1983-1987) dans les zones sahéliennes, avec de petits barrages et des digues filtrantes. Les bas-fonds soudaniens font l'objet d'un intérêt accru depuis une vingtaine d'années et de nombreux aménagements rizicoles ont été réalisés par différents projets de développement. Avec le changement climatique, ils sont perçus comme des opportunités pour accroître la production agricole et augmenter la résilience des agricultures familiales (Ahmadi et Témé, 1998; Torou et al., 2018). Le Burkina Faso a ainsi engagé une politique volontariste d'aménagement des bas-fonds, dans un objectif d'indépendance alimentaire et de réduction des risques climatiques. 
Les intervenants de développement voient l'aménagement principalement comme une infrastructure physique permettant de modifier les flux hydriques, et ainsi d'améliorer le potentiel productif de l'espace aménagé. Or, tout aménagement rebat les cartes de la répartition des terres et induit des gagnants et des perdants (Bouju, 1998). Le foncier - ensemble des rapports qui s'établissent entre les hommes pour l'accès à la terre et son contrôle (Le Bris et al., 1982) - constitue un enjeu déterminant de l'impact d'un projet d'aménagement, à un triple titre (Lavigne Delville et al., 2000):

- les opportunités économiques créées par l'aménagement ne sont accessibles qu'aux acteurs obtenant une parcelle dans l'espace aménagé, qui ne sont en général qu'une partie des habitants du village;

- la répartition des gains et des pertes entre les ménages et les individus - et donc l'impact du projet en termes de lutte contre la pauvreté et de résilience des ménages - dépend des règles de répartition des nouvelles parcelles, et de la façon dont ces règles recomposent les droits préexistants ;

- faute de prendre en compte les droits existants et les enjeux politiques et fonciers de l'aménagement, de nombreuses interventions suscitent ou réveillent des conflits fonciers parfois graves, et qui peuvent bloquer la mise en culture de l'aménagement.

Pourtant, plus de vingt ans après les premiers aménagements, le constat reste le même sur les projets de développement rural : « beaucoup de ces projets n'ont pas d'objectifs en matière foncière, mais se heurtent tout de même, chemin faisant, à des contraintes foncières. En général, ceux-ci ont alors tendance à fermer les yeux sur ces questions réputées brûlantes, même lorsqu'elles sont bien identifiées » (Sanou, 2015). Après des travaux à la fin des années 1990, la recherche ne s'y est ellemême guère intéressée.

En étudiant les enjeux fonciers de l'aménagement de Lofing, cet article se propose de contribuer à la réflexion sur les impacts des stratégies d'intervention d'aménagement de basfonds, en termes de recompositions foncières et de conflits. Lofing est un village dagara situé à $7 \mathrm{~km}$ au nord-est de Dano dans la province du Ioba, au sud-ouest du Burkina Faso. De nombreux bas-fonds y ont été aménagés par différents projets. Le territoire de Lofing est traversé par un bas-fond de plus de $3 \mathrm{~km}$ de long (Dorée, 2017), dont 21 hectares ont été aménagés (en trois tranches, entre 2014 et 2016) pour la culture du riz par la fondation allemande Dreyer. C'était sa première expérience dans ce domaine. L'aménagement est constitué d'une digue amont, d'un drain central surcreusé permettant d'évacuer les excès d'eau et pouvant être fermé pour irriguer les parcelles par débordement, de deux canaux latéraux, et d'un casiérage des parcelles. Il a été choisi après un diagnostic régional comme site de recherche par GENERIA, projet de recherches pluridisciplinaires visant à améliorer les performances des aménagements. Cet article résulte d'enquêtes qualitatives (entretiens individuels formels et informels, observation participante) menées en continu de septembre à décembre 2017 dans le cadre du projet.

Partant du constat qu'un aménagement s'inscrit dans un espace déjà approprié, nous commencerons par analyser les règles et modalités d'accès à la terre (et de contrôle de cet accès) dans les bas-fonds en zone non aménagée, en les resituant dans l'histoire du peuplement du village. Nous identifierons ensuite les recompositions foncières induites par l'aménagement, en analysant son histoire, sa localisation dans les trames foncières lignagères, la politique du projet en matière de foncier et les modes concrets d'attribution des parcelles. Nous mettrons ainsi en évidence les effets redistributifs de l'aménagement.

\section{Les enjeux fonciers des aménagements de bas-fonds: un cadre analytique}

En Afrique, les droits sur la terre relèvent de normes sociales structurées autour de l'histoire du peuplement et du statut social des acteurs (Berry, 1989). L'histoire du peuplement construit à la fois des territoires et des communautés politiques (Chauveau et al., 2004; Kuba et al., 2004). Un bas-fond n'est pas qu'un espace physique, c'est aussi un espace socialisé et approprié, où se construisent et se déconstruisent les relations sociales:

- il s'inscrit dans des territoires politiques et dans des patrimoines lignagers et familiaux;

- il fait fréquemment l'objet d'usages multiples, régis chacun par des règles foncières spécifiques.

À des degrés variables, un aménagement hydroagricole recompose ces droits et les territoires. La façon dont les droits antérieurs ont été traités, le degré de réorganisation foncière induite par l'aménagement, la façon dont sont définies les nouvelles règles d'accès à l'espace aménagé ont des impacts très forts sur la répartition entre les différents acteurs des gains et des pertes induits par l'aménagement. Un impact social et économique positif suppose que les perdants soient peu nombreux ou puissent bénéficier de compensations jugées satisfaisantes, et que les ménages les plus vulnérables aient accès au bas-fond aménagé et soient en mesure d'exploiter leur parcelle de façon efficace.

Les nouvelles règles d'accès à l'aménagement peuvent être liées à des normes légales, avoir été imposées par le projet qui crée ainsi son propre droit (Roth, 2009), ou avoir été négociées au village. Mais ce qui compte ne sont pas tant les règles officielles que les «normes pratiques» (Olivier de Sardan, 2010) qui président à la distribution des parcelles.

\section{Histoire du peuplement, patrimoines lignagers et accès à la terre des bas-fonds à Lofing}

\subsection{Histoire du peuplement, quartiers et patrimoines lignagers à Lofing}

La région de Dano fait partie de l'aire d'expansion des Dagara, venus du Ghana, qui ont progressivement colonisé le sud du Burkina Faso au XIX ${ }^{\mathrm{e}}$ siècle (Hiën, 2000 ; Kuba et al., 2001), en repoussant les populations installées antérieurement (Bwaba puis Puguli). Le village de Lofing est constitué de trois lignages autochtones Dagara (on parle localement de «clans»), qui ont fondé les trois quartiers les plus anciens: les Gnuolè ont fondé Ourgan, les Birfuorè Yèrègan, les Kpièlè Yiziri. Bien qu'arrivés les premiers, les Gnuolè n'ont qu'une petite portion du territoire. Des membres de trois autres lignages ont ensuite été accueillis par les lignages autochtones, 


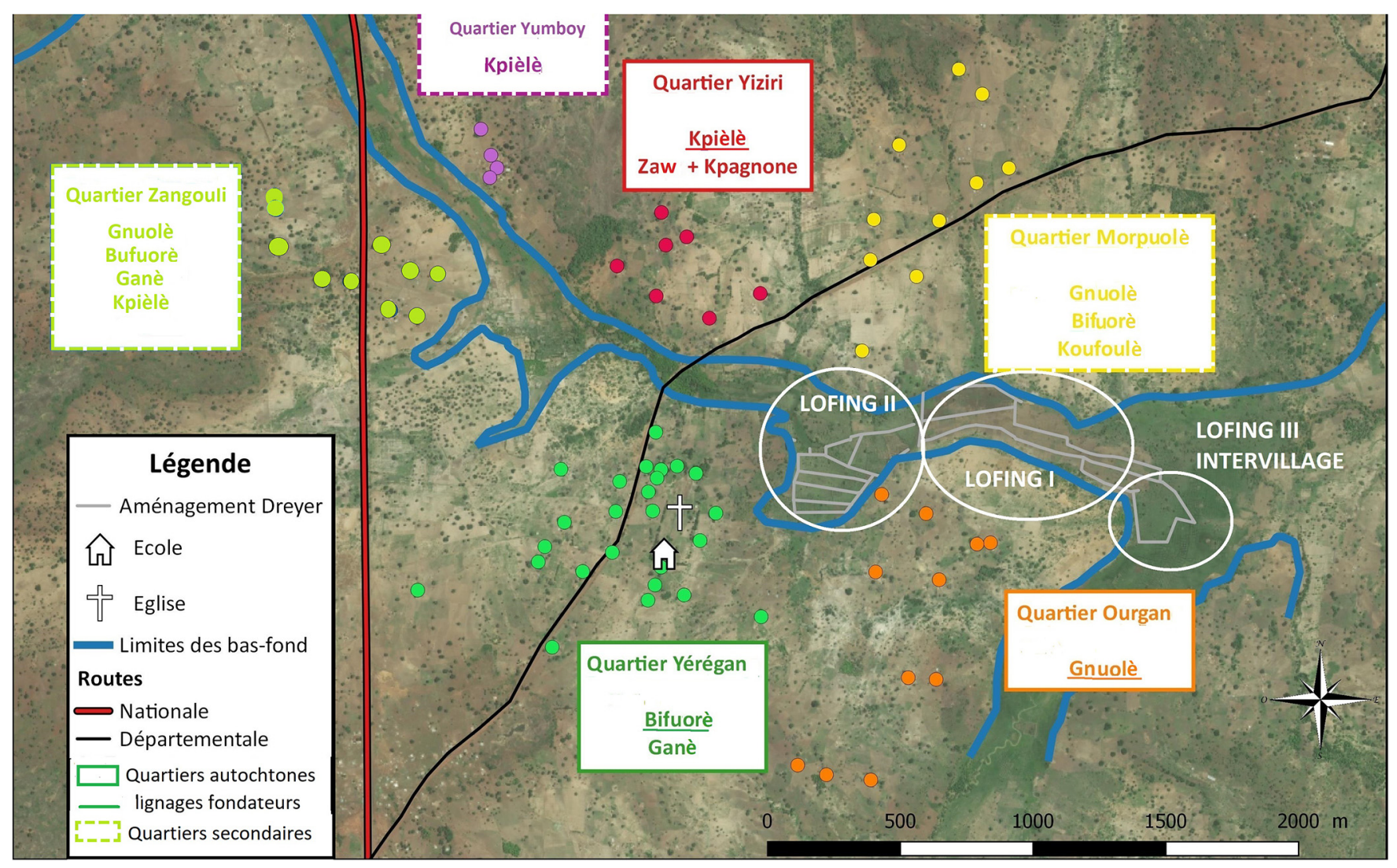

Fig. 1. Le territoire de Lofing, les quartiers et les «clans» (élaboration : J. Robin, sur fond de carte d'A. Dorée).

Fig. 1. The Lofing territory, the hamlets and the lineages.

parfois dans de nouveaux quartiers: Mopuèlè, installé sur les terres des Gnuolè ; Zangouli, sur les terres des Birfuorè, et Yumboy, sur les terres des Kpièlè (Fig. 1). Au fil d'une histoire complexe, le pouvoir rituel a changé plusieurs fois de lignage. C'est aujourd'hui le lignage Gnuolè (quartier Ourgan) qui détient la fonction de maître de terre, et les Birfuorè (quartier Yèrègan) leur disputent la maîtrise du couteau sacrificiel, indispensable aux rituels (Somé, s.d.).

\subsection{Patrimoines fonciers lignagers, ménages et exploitations agricoles}

Au fil de l'histoire, en fonction de la démographie et des scissions, les patrimoines lignagers constitués par la défriche ont été étendus et partagés entre les différentes «maisons » (yiè) du lignage. Le yir (singulier de yiè) désigne à la fois l'unité d'habitation, mais aussi le sous-lignage et l'unité de patrimoine foncier. Les grandes concessions regroupant trois générations ont laissé place à des exploitations agricoles regroupant un ou plusieurs ménages apparentés, disposant de droits de culture permanents sur une portion du patrimoine familial (Pradeau, 1970). Le yir (au sens large) demeure une unité foncière, mais peut regrouper aujourd'hui plusieurs maisons, plusieurs exploitations agricoles différentes. Celles-ci regroupent un ou plusieurs ménages apparentés, et se recomposent en fonction de nombreux facteurs comme la main-d'œuvre, la disponibilité en terre, les types de production, les relations et l'entente intrafamiliale, les migrations ou le statut de chaque exploitant.

\subsection{Un accès fluide à la terre, au sein et en dehors des groupes familiaux}

Les exploitants agricoles disposent de droits d'exploitation permanents sur les portions du patrimoine lignager héritées de leur père. Ils peuvent également solliciter des droits d'usage sur d'autres patrimoines, au sein du même lignage ou auprès d'un autre. L'accès est facilité lorsque des relations de parenté sont en jeu. Même si la transmission des droits sur la terre est patrilinéaire, un individu peut aisément demander une parcelle dans sa famille maternelle. Il n'y a pas de ventes et très peu de locations. De nombreux prêts assurent une circulation des droits de culture. Une contribution, même en nature, est rarement exigée, mais l'emprunteur a des obligations sociales envers son tuteur. Sous peine de voir son droit remis en cause, il se doit d'entretenir de bonnes relations, en l'invitant au cabaret de dolo (bière de mil), en lui offrant des fruits de son jardin ou une tine (bassine) de sa récolte, etc. La souplesse de l'accès aux droits de culture permet des ajustements entre dotations et besoins en terre.

\subsection{Contrôle lignager et accès à la terre dans les bas- fonds}

Le bas-fond est intégré dans les patrimoines lignagers : les portions de bas-fonds proches d'un quartier sont principalement attribuées aux membres du lignage fondateur du quartier. La mise en culture dépend des conditions hydriques et des 


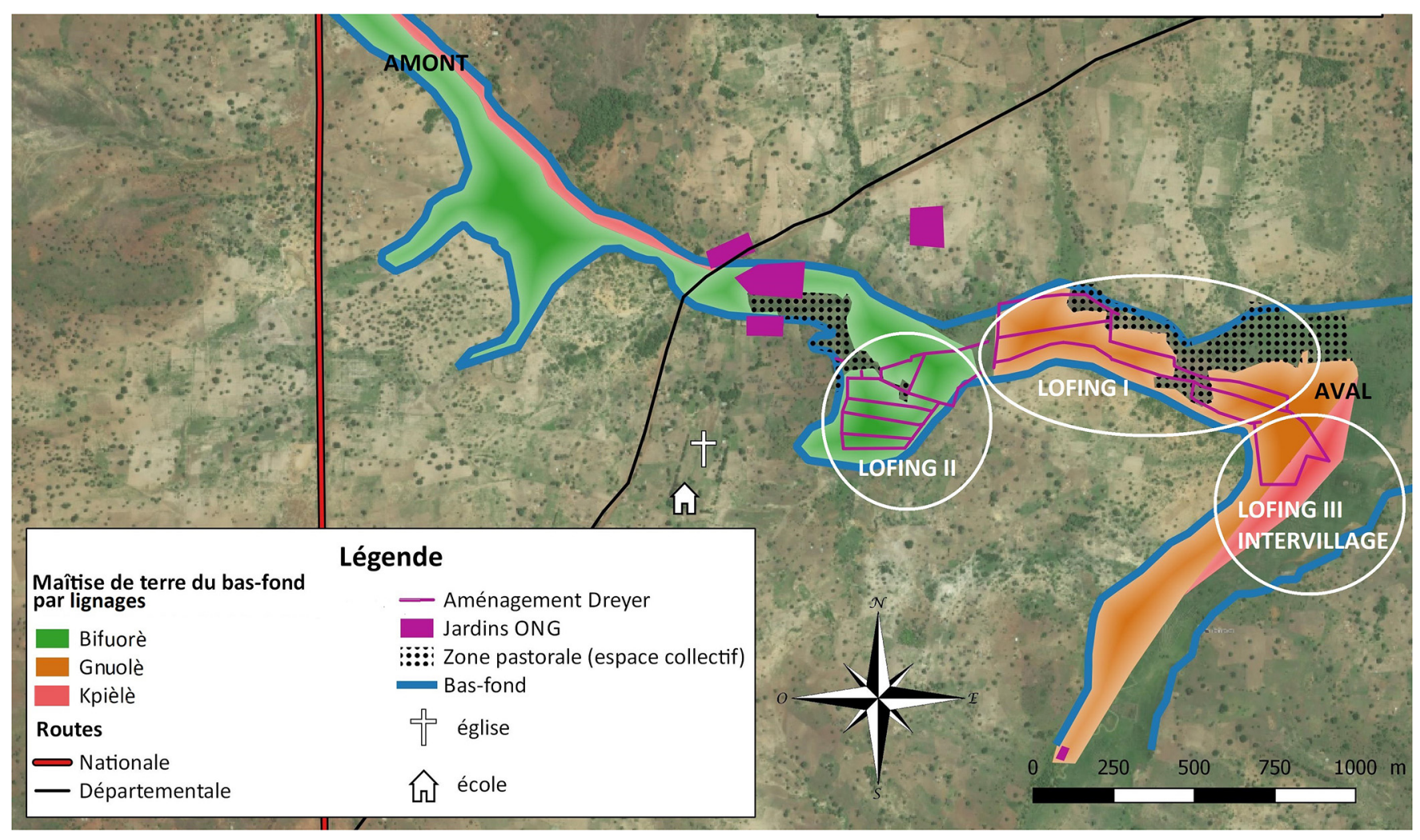

Fig. 2. Maîtrises foncières lignagères et emprise de l'aménagement (élaboration: Justine Robin sur image de l'aménagement réalisée par A. Dorée).

Fig. 2. The lineage land masterships and the area covered by the scheme.

intérêts économiques. L'exploitation en saison des pluies (riz, buttes avec cultures associées) ou en saison sèche (planches maraîchères) est concentrée dans la partie amont, moins inondée, principalement contrôlée par les Birfuorè. Tous les habitants de Lofing qui le souhaitent ont accès et cultivent sur le bas-fond, même si ce n'est pas nécessairement sur les terres de leur lignage, ou au sein de la localité de Lofing. Les clans allochtones ont accès au bas-fond par des droits délégués par les lignages fondateurs. La partie aval du bas-fond, principalement contrôlée par les Gnuolè, était autrefois cultivée en buttes de maïs. Elle est en grande partie abandonnée depuis plusieurs décennies et sert de pâturage de saison sèche, mais les droits correspondants n'ont pas disparu.

\section{L’aménagement, une redistribution des cartes}

L'aménagement redéfinit le parcellaire et redistribue les droits sur la terre aménagée en fonction de critères nouveaux. Dans les règles définies par la fondation Dreyer, c'est l'adhésion au groupement (concrétisée par le paiement d'une cotisation de 1000 CFA) et le fait d'avoir participé au chantier de l'aménagement qui donnent droit à une parcelle (d'une surface de $2000 \mathrm{~m}^{2}$ pour les hommes et $1000 \mathrm{~m}^{2}$ pour les femmes). Pour assurer une équité entre les membres, les parcelles - dont la qualité varie selon la localisation - devaient être tirées au sort entre les membres, sur place, à la fin du chantier. Mais les listes d'adhérents n'ont pas été totalement tenues à jour, le suivi de la participation au chantier encore moins. Faute de plan d'aménagement, la Fondation ne savait pas à l'avance combien il y aurait de parcelles et si «l'offre» correspondrait à la «demande».

\subsection{Le conflit lors de la distribution des parcelles: un sentiment de spoliation de la part des possesseurs de l'espace aménagé}

La distribution des parcelles a été marquée par un conflit avec les anciens propriétaires. Aucune mesure spécifique n'était au départ prévue pour ceux qui possédaient des terres sur l'espace aménagé, d'autant que cette partie du bas-fond était abandonnée depuis plusieurs décennies (Dorée, 2017). La fondation Dreyer avait demandé que les détenteurs coutumiers concernés cèdent leurs terres au village, pour son intérêt collectif. Un acte de donation a apparemment été établi, dont nous n'avons pu retrouver trace. Lofing I empiète principalement sur les terres des Gnuolè (Fig. 2). De nombreux Birfuorè ont adhéré au groupement, tandis que des Gnuolè, ayant eu le sentiment de s'être fait forcer la main, ont refusé de participer aux discussions et n'ont pas adhéré. C'est au moment de la distribution des parcelles que les Gnuolè de Ourgan ont pris conscience que les attributaires des autres quartiers gagnaient une nouvelle parcelle rizicole, sans rien perdre de leurs terres sur la partie non aménagée, alors qu'euxmêmes perdaient le contrôle de leurs terres sans compensation. Ce sentiment de spoliation a été d'autant plus fort que le 
groupement est dirigé par des Birfuorè. En effet, c'est l'un d'entre eux qui avait fait la démarche auprès de la fondation pour obtenir un aménagement au village. L'exclusion de l'accès aux parcelles (faute d'avoir adhéré) se doublait ainsi d'une dépossession symbolique et politique sur l'espace aménagé et le contrôle de l'aménagement.

\subsection{Des adaptions pragmatiques, facteurs de résolution du conflit mais porteuses d'incompréhension}

Cette situation a provoqué des tensions lors de la distribution des parcelles. Les anciens propriétaires adhérents au groupement ont exigé que leur parcelle soit localisée sur leurs terres familiales. Des anciens propriétaires qui n'avaient pas cotisé ont réclamé une parcelle. La Fondation a dû changer les règles pour leur donner satisfaction. Mais cela a augmenté le nombre d'attributaires, qui a dépassé le nombre de parcelles disponibles, obligeant à subdiviser les parcelles non encore attribuées, et même à redécouper certaines parcelles qui venaient d'être attribuées dans la partie amont. Cet arrangement pragmatique, négocié en urgence, a permis de résoudre le conflit, mais a suscité des inégalités entre les attributaires, notamment quant à la taille de la parcelle qu'ils ont reçue (la partie aval étant initialement réservée aux femmes, avec des parcelles de plus petite taille). Il a aussi provoqué un flou sur les règles d'accès, dont la logique est devenue difficilement compréhensible pour les villageois.

Pour résoudre la pénurie de parcelles, l'ONG a aménagé l'année suivante une nouvelle section en aval du premier aménagement (Lofing II). Ces redécoupages, et les réattributions de parcelles qui ont suivi, n'ont pas été compris par les attributaires déplacés qui ont dû réinvestir du travail pour planer leur nouvelle parcelle, donnant le sentiment d'un interventionnisme générateur d'insécurité foncière.

\subsection{L'occupation actuelle, fruit de nombreux ajustements post-aménagement}

Les attributaires ont en principe pour obligation d'exploiter eux-mêmes leur parcelle, toute parcelle non exploitée pouvant être retirée et réaffectée par le groupement. Les enquêtes révèlent cependant de nombreux ajustements par rapport à la distribution initiale :

- dans Lofing I et Lofing II, plusieurs attributaires dont les parcelles étaient sur des terres auparavant contrôlées par des Gnuolè ont été expulsés par ces derniers ;

- de nombreux transferts intra-familiaux, non enregistrés auprès du groupement, ont eu lieu, l'attributaire officiel (homme ou femme) cédant son droit d'exploiter à un conjoint ou à un parent de la même exploitation. En pratique, les parcelles attribuées aux membres d'une exploitation ont été mutualisées en son sein et rentrent dans le jeu des réaffectations internes de droits de culture;

- bien qu'interdits, des prêts ont lieu, le plus souvent entre membres du même yir ou du même lignage (ils sont rares entre personnes de lignage différent);

- des dons ont été faits en faveur des gros agriculteurs. Certains gros agriculteurs (dont des responsables du groupement) cumulent ainsi plusieurs parcelles ;
- malgré l'obligation de mise en valeur, on observe quelques parcelles non exploitées, qui ne font pas l'objet de reprises par le groupement. Beaucoup d'exploitants témoignent d'une difficulté à accéder à une parcelle par le prêt ou la location, même si celle-ci n'est pas cultivée.

La figure 3 montre la répartition actuelle des parcelles en fonction du lignage de l'exploitation à laquelle appartient l'attributaire. Pour les femmes attributaires, c'est généralement l'exploitation de leur mari. La parcelle d'une femme Gnuolè mariée dans un ménage Birfuorè est ainsi marquée en vert, car elle contribue à l'économie d'un ménage Birfuorè. La distribution initiale -qui a permis aux Gnuolè d'obtenir des parcelles là où leurs pères cultivaient, et les a donc concentrés dans la zone amont - et les différents réajustements ultérieurs produisent une répartition actuelle relativement équilibrée entre lignages en nombre de parcelles, moins en surface cumulée. Rares sont les non-autochtones à avoir eu une parcelle (Fig. 3).

\subsection{Les femmes et l'aménagement du bas-fond}

Les femmes pouvaient demander une parcelle, mais de plus petite taille. Sur la soixantaine de femmes demandeuses, une quarantaine exploite actuellement une parcelle dans l'aménagement, le plus souvent en aval de celui-ci (Fig. 4). Le fait d'avoir eu une parcelle attribuée à son nom ne signifie pas qu'elle soit sous son contrôle: dans de nombreux cas, la parcelle attribuée à une femme a été réaffectée à un autre membre de son ménage, voire à un proche parent. De plus, l'attribution d'une parcelle à une femme ne règle pas la question de la transmission de ce droit par héritage. Dans un contexte où la transmission du patrimoine foncier est patrilinéaire, il est probable que, lors du décès d'une attributaire, la parcelle soit intégrée dans le patrimoine familial et transmise selon les règles locales, au risque d'évincer les filles.

Plus largement, le contenu des droits obtenus par les attributaires (hommes et femmes) n'a pas été totalement explicité. Au-delà des différents ajustements observés, les acteurs interrogés ont des avis divergents sur le futur de ces droits : sont-ils définitifs ? Sont-ils transmissibles par héritage? Et si oui, selon quelle règle? Perdureront-ils en cas d'abandon de la parcelle ou de l'aménagement? Les anciens possesseurs ont-ils donné définitivement les terres, retrouveront-ils le contrôle foncier des terres aménagées en cas de disparition du groupement? Cela témoigne d'une intégration encore partielle des parcelles aménagées dans les normes foncières locales. Faute d'avoir été anticipées et négociées, c'est lorsque ces questions se poseront concrètement (par exemple, lors des premiers décès d'attributaires) que la réponse - consensuelle ou conflictuelle - sera définie.

\section{Aménagements et conflits de limites territoriales}

Les enjeux fonciers se croisent avec des enjeux territoriaux. La localisation de l'aménagement est définie sur des critères topographiques et hydrauliques, qui ne correspondent pas nécessairement aux limites territoriales $\mathrm{du}$ village 


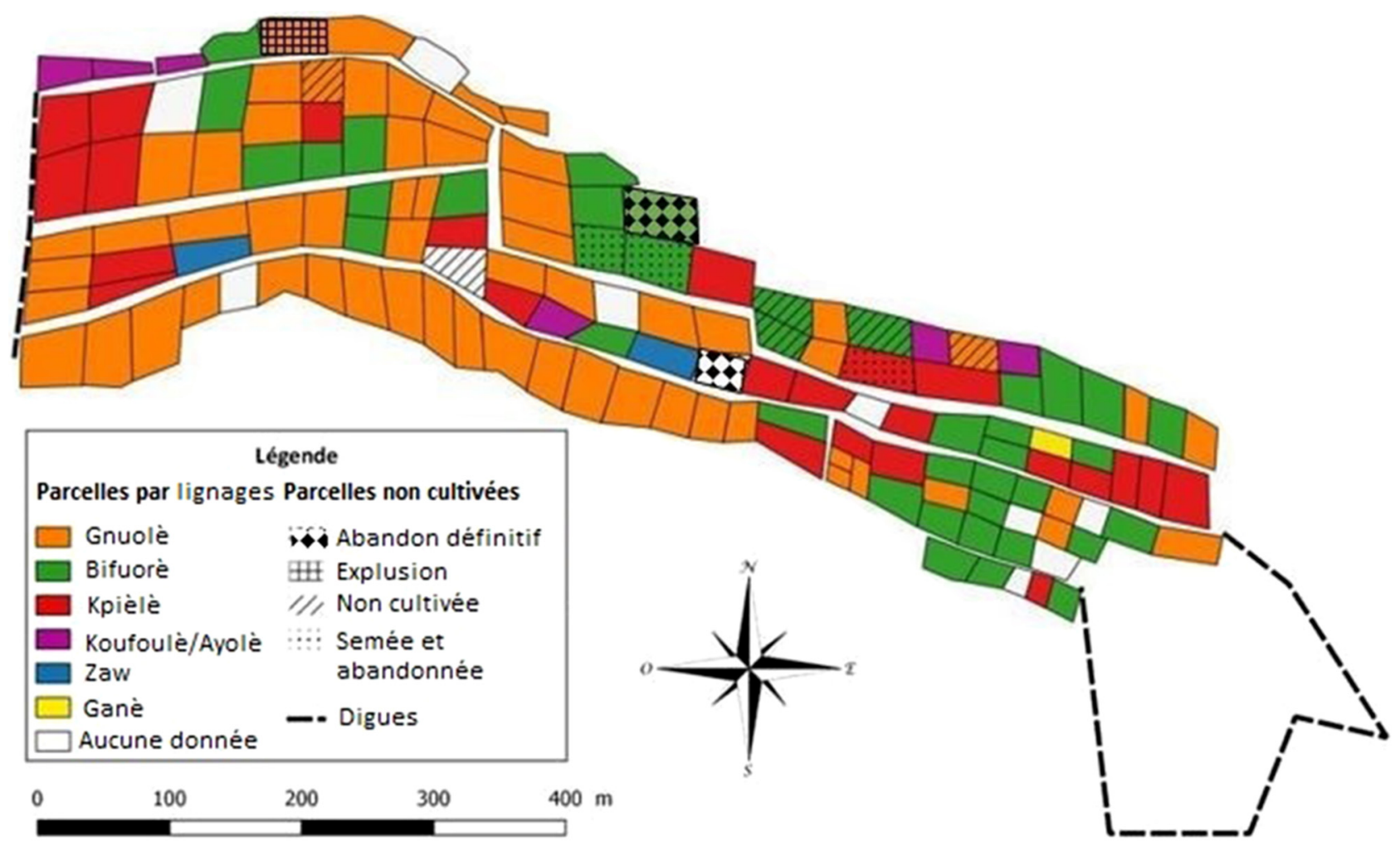

Fig. 3. Répartition actuelle des exploitants de l'aménagement, par lignage (Lofing I) (source: enquêtes, fond de carte: A. Dorée).

Fig. 3. Current distribution of farmers in the scheme, by lineage (Lofing I).

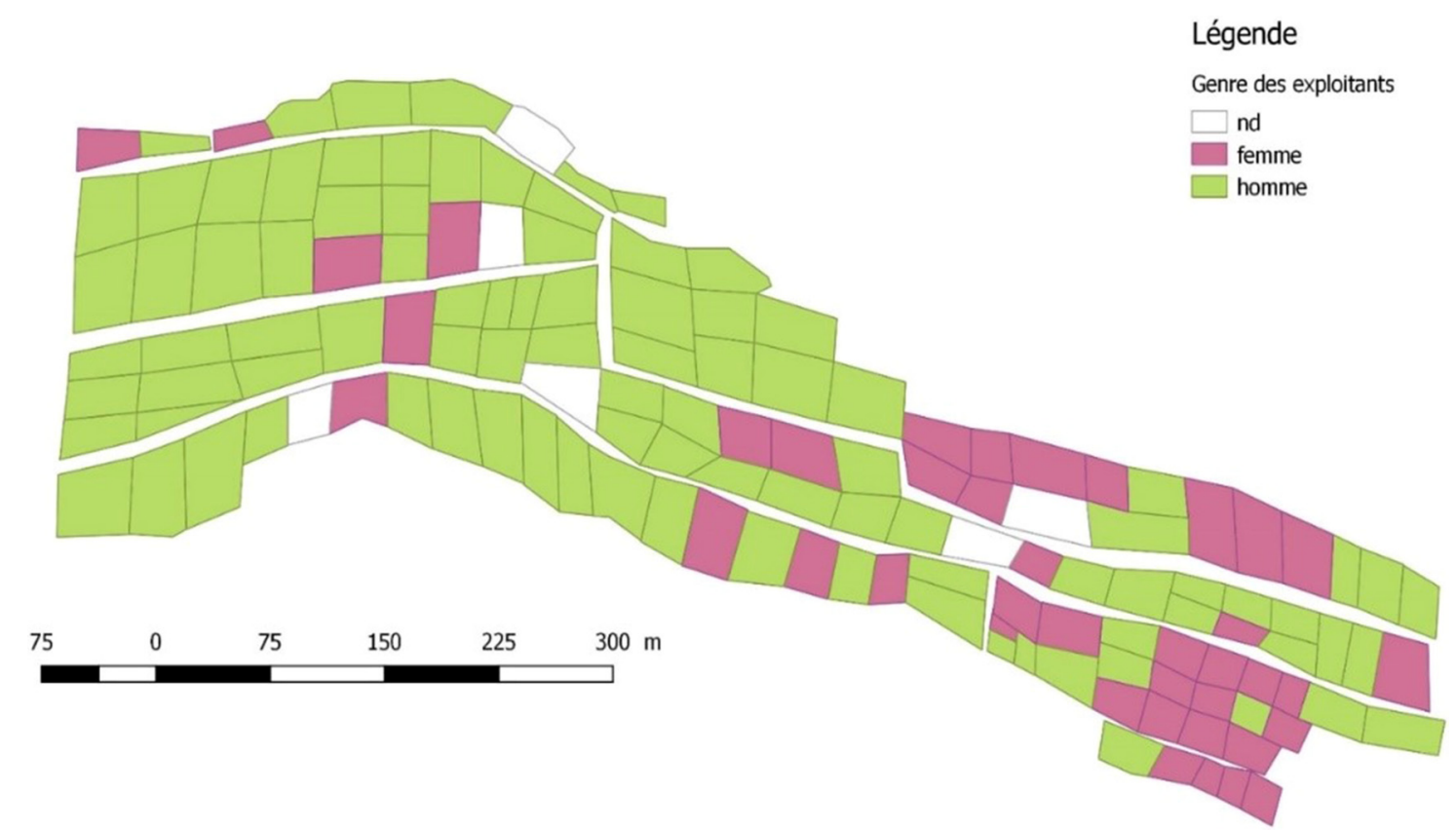

Fig. 4. Répartition actuelle des exploitants par genre (Lofing I) (source: enquêtes, fond de carte: A. Dorée).

Fig. 4. Current distribution of farmers in the scheme, by gender (Lofing I).

concerné. Tout d'abord, le village administratif qui est la base des interventions de développement ne correspond pas toujours au village coutumier (défini par un autel de terre, et différents quartiers et hameaux installés sous l'égide des pouvoirs coutumiers). Les limites des villages administratifs ne sont jamais définies. Tout comme celles des territoires 
coutumiers qui ne sont que rarement établies de façon claire pour laisser la possibilité d'expansion en fonction des besoins. Lorsque les espaces périphériques étaient peu exploités, il n'y avait pas forcément de limite définie. Au carrefour de deux fronts de défriche, on rencontre alors des terroirs imbriqués, exploités par des ressortissants des deux villages.

Un conflit a éclaté lors de l'aménagement par la fondation Dreyer de la partie aval du bas-fond, aux limites entre Lofing et Bavoulé (Lofing III). Cette zone se situe à la rencontre des territoires des Gnuolè de Lofing et des Zaw de Bavoulé. Suite à un conflit ancien au sujet de la terre entre les Gnuolè de Lofing et les Zaw de Bavoulé, un Zaw était mort après avoir tracé les limites entre les deux villages, ce qui constitue une faute irréparable sur le plan coutumier. Puis un Gnuolè avait souhaité cultiver dans la zone litigeuse. Il mourut aussitôt dans son champ. Dès lors, personne n'osa s'aventurer à cultiver dans cette zone. La Fondation n'avait pas identifié cet enjeu, ni pris acte du fait que, les parcellaires dans cette zone étant enchevêtrés, on ne pouvait pas distinguer de limite claire entre les deux territoires. L'aménagement de Lofing III est venu réveiller cette vieille querelle, ravivant les débats au sujet des droits sur l'espace aménagé et la peur de cultiver dans la zone. Malgré la requalification de l'aménagement en « intervillage », l'intégration d'attributaires de Bavoulé et de Lofing, regroupés chacun de chaque côté du canal, et l'intervention des responsables administratifs et coutumiers, il n'a pas été possible de trouver une solution socialement acceptable, ni de lever l'interdit de culture. Par conséquent, cette tranche de l'aménagement n'est que très peu mise en valeur.

\section{Conclusion}

Autant qu'une intervention technique, un aménagement de bas-fonds est un processus social qui met en interaction différents acteurs aux logiques diverses, voire divergentes. Chacune de ses parties prenantes va tenter d'influencer le déroulement de l'action pour l'orienter dans le sens de ses intérêts. C'est l'histoire des interactions, négociations, pratiques autour de l'aménagement qui permet de comprendre comment s'est construit son impact.

Notre étude a mis en évidence les conséquences de l'aménagement sur la répartition des droits entre groupes (villages, clans, yiè), et dévoilé les tensions qui en ont résulté, du fait que des groupes ont vu leurs droits antérieurs remis en cause et s'imposer une redistribution sans véritable négociation ni compensation. Le cas de Lofing montre l'importance des maîtrises foncières lignagères (peu évoquées dans les études antérieures) et le fait que les droits fonciers ne disparaissent pas lorsque l'espace n'est plus exploité depuis de nombreuses années et peuvent être réactualisés à l'occasion de l'aménagement.

La plupart des tensions observées ont été provoqués par l'absence d'anticipation des enjeux, une insuffisance d'information et de communication entre les intervenants du projet et les villageois. Les flous sur les règles ou la façon dont se déroulent les évènements donnent lieu à diverses interprétations. La solution pragmatique trouvée au moment de la distribution des parcelles a permis de gérer les tensions avec les anciens propriétaires et de rendre la distribution socialement acceptable. Mais cela a laissé des traces. De plus, faute d'avoir maîtrisé le processus de répartition des parcelles, le groupement a du mal à gérer les tensions et ces conflits remontent fréquemment à l'aménageur qui n'a pas pour rôle de les traiter. Le contenu des droits accordés aux exploitants est incertain et l'obligation de mise en culture personnelle de la parcelle attribuée est contradictoire avec les dynamiques des unités familiales. Cette étude de cas confirme les deux grandes causes de conflits liés aux aménagements, identifiées près de 20 ans plus tôt (Lavigne Delville et al., 2000): les enjeux politiques et territoriaux de l'aménagement, d'une part, (qui porte le projet et pourquoi ? qui contrôle l'espace à aménager et peut prendre la décision d'aménager?); les enjeux économiques, d'autre part, (qui gagne et qui perd? quels impacts des choix en termes d'accès aux parcelles aménagées ?). Mais elle met plus clairement en avant les enjeux politiques de l'aménagement (entre lignages et entre villages) et de son insertion dans l'histoire foncière du lieu: la question des conditions qui permettent un consentement suffisant sur la réorganisation foncière induite par l'aménagement dépasse la question de l'équité de la répartition des parcelles entre individus. Ces enjeux sont inhérents à tout aménagement. Ils ne débouchent pas nécessairement sur des conflits. Les risques sont plus ou moins vifs selon les configurations. Les conflits peuvent aussi être provoqués ou aggravés par l'intervenant, par sa maladresse ou son ignorance de ces enjeux : s'il est aveugle à ces derniers, il risque d'autant plus d'être instrumentalisé.

Les aménageurs hésitent à s'engager directement dans les négociations foncières pour lesquelles ils manquent de clés de compréhension, et préfèrent laisser «le village» gérer les questions foncières et négocier l'accord des ayants droit, pensant un peu naïvement qu'un accord écrit permet de stabiliser cet accord. Mais les enjeux politiques de l'aménagement, la volonté d'aboutir des responsables du groupement, et les relations asymétriques avec les intervenants peuvent aboutir à des accords de façade cachant des désaccords qui ressortiront à la première occasion. De plus, en définissant de façon normative des règles nouvelles pour l'accès à la terre, ils s'ingèrent en pratique dans le foncier, au risque de créer des situations que les groupements ne peuvent pas gérer et d'être obligés de s'impliquer pour tenter de résoudre les tensions qu'ils ont en partie suscitées.

Lofing était le premier aménagement de bas-fonds réalisé par la fondation Dreyer, qui en a découvert les enjeux à l'occasion. Le cas n'était pas trop problématique. Mais des équipes plus expérimentées n’ont pas nécessairement de démarches plus structurées en termes de foncier. Une analyse comparée des démarches et des savoir-faire des intervenants, d'une part, et une étude comparée sur les dynamiques foncières dans des aménagements anciens et les modes de transmission des parcelles, d'autre part, permettraient de mieux comprendre les modes de transmission des droits. Elles donneraient des outils pour mener des négociations locales pré-aménagement, pour définir des règles légitimes et opératoires d'attribution et de transmission des droits sur les parcelles aménagées.

Remerciements. Cette recherche a été menée dans le cadre du projet GENERIA (Gestion des nouveaux risques et des opportunités des terres inondables pour l'agriculture africaine), financé par le programme AGRICORA du Ministère français des affaires étrangères et piloté par WASCAL (West African Science Service Center on Climate Change and Adapted Land Use). 
Nous remercions sincèrement le Pr Magloire Somé pour ses apports à la compréhension de l'histoire de Lofing, et Georges Serpantié (IRD/GRED) pour son appui.

\section{Références}

Ahmadi N, Témé B (eds). 1998. Aménagement et mise en valeur des bas-fonds au Mali. Montpellier (France) : Cirad.

Berry S. 1989. Social institutions and access to resources in African agriculture. Africa 59(1): 41-55.

Bouju J. 1998. Contrôle foncier et conflits pour les ressources : l'accès aux bas-fonds aménagés comme enjeu de pouvoir local. In: Ahmadi N, ed. Aménagement et mise en valeur des bas-fonds au Mali. Montpellier: Cirad, pp. 95-108.

Chauveau J-P, Jacob J-P, Le Meur P-Y. 2004. L'organisation de la mobilité dans les sociétés rurales du Sud. Gouverner les hommes et les ressources : dynamiques de la frontière. Autrepart 30(2): 3-23. DOI: 10.3917 /autr.030.0003.

Dorée A. 2017. Gérer les nouveaux risques des bas-fonds soudaniens (Dano, Burkina Faso). Montpellier (France) : IRC/SUPAGRO.

Hiën PC. 2000. Frontières et conflits chez les Dagara et leurs voisins au sud-ouest du Burkina Faso (XVIIIième-XIXième siècle). In: Kuba R, Lentz C, Werthmann K, eds. Les Dagara et leurs voisins : histoire de peuplement et relations interethniques au sud-ouest $d u$ Burkina Faso. Frankfurt am Main (Allemagne) : Johann Wolfgang Goethe-Universität, pp. 427-440.

Kuba R, Lentz C, Werthmann K. 2001. Les Dagara et leurs voisins : histoire de peuplement et relations interethniques au sud-ouest du Burkina Faso. Frankfurt am Main (Allemagne) : Johann Wolfgang Goethe-Universität.

Kuba R, Lentz C, Somda CN. 2004. Histoire du peuplement et relations interethniques au Burkina Faso. Paris (France) : Karthala.

Lavigne Delville P. 1998. Logiques paysannes d'exploitation des basfonds en Afrique soudano-sahélienne. In: Ahmadi N, Témé B, eds. Aménagement et mise en valeur des bas-fonds au Mali, bilan et perspectives nationales, intérêt pour la zone de savane ouestafricaine. Montpellier (France) : CIRAD, pp. 77-93.

Lavigne Delville P, Bouju J, Le Roy E. 2000. Prendre en compte les enjeux fonciers dans une démarche d'aménagement: stratégies foncières et bas-fonds au Sahel. Paris (France) : GRET.

Le Bris É, Le Roy É, Leimdorfer F, Grégoire E. 1982. Enjeux fonciers en Afrique noire. Paris (France): Orstom/Karthala.

Olivier de Sardan J-P. 2010. Développement, modes de gouvernance et normes pratiques (une approche socio-anthropologique). Canadian Journal of Development Studies/Revue canadienne d'études du développement 31(1-2): 5-20. DOI: 10.1080/ 02255189.2010 .9669327$.

Pradeau C. 1970. Kokolibou (Haute-Volta) ou le pays Dagari à travers un terroir. Études rurales 37-39: 85-112.

Raunet M. 1985. Bas-fonds et riziculture en Afrique. Approche structurale comparative. L'Agronomie tropicale 40(3): 181-201.

Roth D. 2009. Vaincus au tribunal, gagnants sur le terrain. Réforme foncière en Indonésie, entre droit coutumier, droit du projet et droit étatique. In: Colin J-P, Léonard E, Le Meur P-Y, eds. Les politiques d'enregistrement des droits fonciers. Du cadre légal aux pratiques locales. Paris (France) : Karthala, pp. 497-530.

Sanou S. 2015. Projet de développement rural : attention au foncier! Des fiches pédagogiques pour comprendre, se poser de bonnes questions et agir sur le foncier en Afrique de l'Ouest. Paris (France) : Comité technique Foncier et Développement. Disponible sur http://www.foncier-developpement.fr/publication/projets-deve loppement-rural-attention-au-foncier/.

Somé M. s.d. Petit précis d'histoire de Lofing. Non publié.

Torou B, Bossa AY, Yameogo T, Yira Y, Da ED, Serpantié G. 2018. Exploitation agricole des bas-fonds : diversité des situations, contraintes et opportunités associées. In: WASCAL, ed. Projet "Gestion des nouveaux risques et des opportunités des terres Inondables pour l'Agriculture africaine (GENERIA)». Rapport scientifique intermédiaire. Ougadougou (Burkina Faso): WASCAL, pp. 22-26.

Citation de l'article : Lavigne Delville P, Robin J. 2019. Aménagement de bas-fonds, politique de l'aménageur et recompositions foncières. Le cas de Lofing au Burkina Faso. Cah. Agric. 28: 18. 